\title{
Geodesic $r$-preinvex functions on Riemannian manifolds
}

Meraj Ali Khan ${ }^{1 *}$, Izhar Ahmad ${ }^{2}$ and Falleh R Al-Solamy ${ }^{3}$

${ }^{*}$ Correspondence: meraj79@gmail.com

'Department of Mathematics, University of Tabuk, Tabuk, Saudi Arabia

Full list of author information is available at the end of the article

\begin{abstract}
In this article, we introduce a new class of functions called $r$-invexity and geodesic $r$-preinvexity functions on a Riemannian manifolds. Further, we establish the relationships between $r$-invexity and geodesic $r$-preinvexity on Riemannian manifolds. It is observed that a local minimum point for a scalar optimization problem is also a global minimum point under geodesic $r$-preinvexity on Riemannian manifolds. In the end, a mean value inequality is extended to a Cartan-Hadamard manifold. The results presented in this paper extend and generalize the results that have appeared in the literature.
\end{abstract}

MSC: 58E17; 90C26

Keywords: invex sets; preinvex functions; $r$-invexity; Riemannian manifolds

\section{Introduction}

Convexity is one of the most frequently used hypotheses in optimization theory. It is well known that a local minimum is also a global minimum for a convex function. A significant generalization of convex functions is that of an invex function introduced by Hanson [1]. Hanson's initial results inspired a great deal of subsequent work, which has greatly expanded the role and applications of invexity in non-linear optimization and other branches of pure and applied sciences.

Ben-Israel and Mond [2] introduced a new generalization of convex sets and convex functions, Craven [3] called them invex sets and preinvex functions, respectively. Jeyakumar [4] studied the properties of preinvex functions and their role in optimization and mathematical programming. Jeyakumar and Mond [5] introduced a new class of functions, namely $V$-invex functions, and established sufficient optimality criteria and duality results in the multiobjective programming problems. Antczak [6] introduced the concept of $r$-invexity and $r$-preinvexity in mathematical programming. Making a step forward Antczak [7] introduced the concept of $V-r$-invexity for differentiable multiobjective programming problems, which is a generalization of $V$-invex functions [5] and $r$-invex functions [6].

On the other hand, in the last few years, several important concepts of non-linear analysis and optimization problems have been extended from Euclidean space to a Riemannian manifolds. In general, a manifold is not a linear space, but naturally concepts and techniques from linear spaces to Riemannian manifold can be extended. Rapcsak [8] and Udriste [9] considered a generalization of convexity, called geodesic convexity, and extended many results of convex analysis and optimization theory to Riemannian manifolds.

\section{空 Springer}

C2014 Khan et al.; licensee Springer. This is an Open Access article distributed under the terms of the Creative Commons Attribution License (http://creativecommons.org/licenses/by/2.0), which permits unrestricted use, distribution, and reproduction in any medium, provided the original work is properly cited. 
The notion of invex functions on Riemannian manifolds was introduced by Pini [10] and Mititelu [11], and they investigated its generalization. Barani and Pouryayevali [12] introduced the geodesic invex set, geodesic $\eta$-invex function, and geodesic $\eta$-preinvex functions on a Riemannian manifold and found some interesting results. Further, Agarwal et al. [13] generalized the notion of geodesic $\eta$-preinvex functions to geodesic $\alpha$-preinvex functions. Recently, Zhou and Huang [14] introduced the concept of roughly $B$-invex set and functions on Riemannian manifolds.

Motivated by work of Barani and Pouryayevali [12] and Antczak [6, 7], we introduce the concept of geodesic $r$-preinvex functions and $r$-invex functions on Riemannian manifolds, which is a generalization of preinvexity as defined in $[6,12]$. Some relations between $r$-invex and geodesic $r$-preinvex functions are investigated. The existence conditions for global minima of these functions under proximal subdifferential of lower semicontinuity are also explored. In the end, a mean value inequality is also derived.

\section{Preliminaries}

In this section we recall some basic definitions and some basic results of Riemannian manifolds, for further study these materials are available in (cf. [15]).

Let $M$ be a $C^{\infty}$-manifold modeled on a Hilbert space $H$, either finite or infinite dimensional, endowed with a Riemannian metric $g_{p}$ on a tangent space $T_{p} M$. The corresponding norm is denoted by \|\|$_{p}$ and the length of a piecewise $C^{1}$ curve $\gamma:[a, b] \rightarrow M$ is defined by

$$
L(\gamma)=\int_{a}^{b}\left\|\gamma^{\prime}(t)\right\|_{\gamma(t)} d t
$$

For any point $p, q \in M$, we define

$$
d(p, q)=\inf \left\{L(\gamma) \mid \gamma \text { is a piecewise } C^{1} \text { curve joining } p \text { and } q\right\},
$$

then $d$ is a distance which induces the original topology on $M$. We know that on every Riemannian manifold there exists exactly one covariant derivative called a Levi-Civita connection, denoted by $\nabla_{X} Y$, for any vector fields $X, Y \in T M$; we also recall that a geodesic is a $C^{\infty}$-smooth path $\gamma$ whose tangent is parallel along the path $\gamma$, that is, $\gamma$ satisfies the equation $\nabla_{d \gamma(t) / d t} d \gamma(t) / d t=0$. Any path $\gamma$ joining $p$ and $q$ in $M$ such that $L(\gamma)=d(p, q)$ is a geodesic and is called a minimal geodesic. The existence theorem for ordinary differential equation implies that for every $v \in T M$, there exist an open interval $J(v)$ containing 0 and exactly one geodesic $\gamma_{v}: J(v) \rightarrow M$ with $d \gamma_{v}(0) / d t=v$. This implies that there is an open neighborhood $\bar{T} M$ of the submanifold $M$ of $T M$ such that for every exp : $\bar{T} M \rightarrow M$ is there is defined $\exp (v)=J_{v}(1)$ and the restriction of exp to a fiber $T_{p} M$ in $\bar{T} M$ is denoted by $\exp _{p}$ for every $p \in M$. We use parallel transport of vectors along the geodesic. Recall that for a given curve $\gamma: I \rightarrow M$, a number $t_{0} \in I$, and a vector $v_{0} \in T_{\gamma\left(t_{0}\right)} M$, there exists exactly one parallel vector field $V(t)$ along $\gamma(t)$ such that $V\left(t_{0}\right)=v_{0}$. Moreover, the mapping defined by $v_{0} \mapsto V(t)$ is a linear isometry between the tangent spaces $T_{\gamma\left(t_{0}\right)} M$ and $T_{\gamma(t)} M$, for each $t \in I$. We denote this mapping by $P_{t_{0}, \gamma}^{t}$ and we call it the parallel translation from $T_{\gamma\left(t_{0}\right)} M$ to $T_{\gamma(t)} M$ along the curve $\gamma$.

If $f$ is a differentiable map from the manifold $M$ to manifold $N$, then $d f_{x}$, denotes the differential of $f$ at $x$. We also recall that a simply connected complete Riemannian manifold of non-positive sectional curvature is called a Cartan-Hadamard manifold. 


\section{Geodesic $r$-invex functions}

In this section, we define geodesic $r$-invex functions and $r$-preinvex functions. Barani and Pouryayevali [12] define the invex sets as follows.

Definition 3.1 Let $M$ be a Riemannian manifold and $\eta: M \times M \rightarrow T M$ such that for every $x, y \in M, \eta(x, y) \in T_{y} M$. A non-empty subset $S$ of $M$ is said to be a geodesic invex set with respect to $\eta$ if for every $x, y \in S$, there exists a unique geodesic $\gamma_{x, y}:[0,1] \rightarrow M$ such that

$$
\gamma_{x, y}(0)=y, \quad \gamma_{x, y}^{\prime}(0)=\eta(x, y), \quad \gamma_{x, y}(t) \in S
$$

for all $t \in[0,1]$.

Remark 3.1 [12] If we consider $M$ to be a Cartan-Hadamard manifold (either infinite or finite dimensional), then on $M$ there exists a natural map $\eta$ playing the role of $x-y$ in the $R^{n}$. Indeed we define the function $\eta$ as

$$
\eta(x, y)=\gamma_{x, y}^{\prime}(0)
$$

for all $x, y \in M$. Here $\gamma_{x, y}$ is the unique minimal geodesic joining $y$ to $x$ (see [16, p.253]) as follows:

$$
\gamma_{x, y}(t)=\exp _{y}\left(t \exp _{y}^{-1} x\right)
$$

for all $t \in[0,1]$. Therefore, every geodesic convex set $S \subseteq M$ is a geodesic convex set with respect to $\eta$ defined in above equation. The converse is not true in general.

Example 3.1 [12] Let $M$ be a Cartan-Hadamard manifold and $x_{0}, y_{0} \in M, x_{0} \neq y_{0}$. Let $B\left(x_{0}, r_{1}\right) \cup B\left(y_{0}, r_{2}\right)=\phi$ for some $0<r_{1}, r_{2}<\frac{1}{2} d\left(x_{0}, y_{0}\right)$, where $B(x, r)=\{y \in M \mid d(x, y)<r\}$ is an open ball with center $x$ and radius $r$. We define

$$
S=B\left(x_{0}, r_{1}\right) \cup B\left(y_{0}, r_{2}\right),
$$

then $S$ is not a geodesic convex set because every geodesic curve passing through $x_{0}$ and $y_{0}$ does not completely lie in $S$. Now we define the function $\eta: M \times M \rightarrow T M$ such that

$$
\eta(x, y)= \begin{cases}\exp _{y}^{-1} x & \text { if } x, y \in B\left(x_{0}, r_{1}\right) \text { or } x, y \in B\left(x_{0}, r_{2}\right), \\ 0_{y} & \text { otherwise }\end{cases}
$$

For every $x, y \in M$, consider $\gamma:[0,1] \rightarrow M$ defined by

$$
\gamma_{x, y}(t)=\exp (t \eta(x, y))
$$

for all $t \in[0,1]$.

Hence $\gamma_{x, y}(0)=y, \gamma_{x, y}^{\prime}(0)=\eta(x, y)$. Barani and Pouryayevali [12] showed that $S$ is a geodesic invex set with respect to $\eta$.

Let $S$ be a geodesic convex subset of a finite dimensional Cartan-Hadamard manifold $M$ and $x \in M$, then there exists a unique point $p_{s}(x) \in S$ such that for each $y \in S, d\left(x, p_{s}(x)\right) \leq$ $d(x, y)$. The point $p_{s}(x)$ is called the projection of $x$ onto $S$ (see [16, p.262]). 
Definition 3.2 [12] Let $M$ be an $n$-dimensional Riemannian manifold and $S$ be an open subset of $M$ which is geodesic invex set with respect to $\eta: M \times M \rightarrow T M$. Let $f$ be a real valued function such that $f: S \rightarrow R$. Then $f$ is said to be an $\eta$-invex function with respect to $\eta$ if

$$
f(x)-f(y) \geq d f_{y}(\eta(x, y))
$$

for all $x, y \in S$.

Definition 3.3 [12] Let $M$ be a Riemannian manifold and $S \subseteq M$ be a geodesic $\eta$-invex set with respect to $\eta: M \times M \rightarrow T M$. The function $f: S \rightarrow R$ is said to be geodesic $\eta$-preinvex if for any $x, y \in S$

$$
f\left(\gamma_{x, y}(t)\right) \leq t f(x)+(1-t) f(y)
$$

for all $t \in[0,1]$, where $\gamma_{x, y}$ is the unique geodesic defined in Definition 3.1. If the above inequality is strict, then $f$ is called a strictly geodesic preinvex function.

Now we define an $r$-invex function and a geodesic $r$-preinvex function on $M$.

Definition 3.4 Let $M$ be a Riemannian manifold and $S \subseteq M$ be a geodesic invex set with respect to $\eta: M \times M \rightarrow T M$. Let $f$ be a real differentiable function $S$. Then $f$ is said to be $r$-invex with respect to $\eta$ if

$$
\begin{aligned}
& \frac{1}{r} e^{r f(x)}-\frac{1}{r} e^{r f(y)} \geq e^{r f(y)} d f_{y}(\eta(x, y)) \quad \text { if } r \neq 0, \\
& f(x)-f(y) \geq d f_{y}(\eta(x, y)) \quad \text { if } r=0 .
\end{aligned}
$$

Definition 3.5 Let $M$ be a Riemannian manifold and $S \subseteq M$ be a geodesic invex set with respect to $\eta: M \times M \rightarrow T M$. The function $f: S \rightarrow R$ is said to be geodesic $r$-preinvex if for any $x, y \in S$, we have

$$
f\left(\gamma_{x, y}(t)\right) \leq \begin{cases}\log \left(t e^{r f(x)}+(1-t) e^{r f(y)}\right)^{\frac{1}{r}} & \text { if } r \neq 0 \\ t f(x)+(1-t) f(y) & \text { if } r=0\end{cases}
$$

If the above inequality is strict, then $f$ is called a strictly geodesic $r$-preinvex function. We give the following non-trivial example for a geodesic $r$-preinvex function that is yet not geodesic $\eta$-preinvex.

Example 3.2 Let $M=\left\{e^{i \theta}: 0<\theta<1\right\}$ and $f: M \rightarrow R$ defined by $f\left(e^{i \theta}\right)=\cos \theta$ with $x, y \in M$, $x=e^{i \alpha}$ and $y=e^{i \beta}$. If $\gamma_{x, y}(t)=e^{i((1-t) \beta+t \alpha)}$ then $f$ is a geodesic $r$-preinvex function but not a geodesic $\eta$-preinvex function at $\alpha=\frac{\pi}{2}, \beta=\frac{\pi}{4}$, since $\cos \left[\frac{\pi}{4}+\frac{\pi}{4} t\right]>\frac{t}{\sqrt{2}}$ at $t=0$.

Proposition 3.1 Iff $: S \rightarrow R$ is a geodesic r-preinvex function with respect to $\eta: S \times S \rightarrow$ $T M$ and $y \in S$, then for any real number $\lambda \in R$, the level set $S_{\lambda}=\{x \mid x \in S, f(x) \leq \lambda\}$ is a geodesic invex set. 
Proof For any $x, y \in S_{\lambda}$ and $0 \leq t \leq 1$, we have $f(x) \leq \lambda, f(y) \leq \lambda$. Since $f$ is geodesic $r$-preinvex function, then we have

$$
f\left(\gamma_{x, y}(t)\right) \leq \log \left(t e^{r f(x)}+(1-t) e^{r f(y)}\right)^{\frac{1}{r}}
$$

or

$$
\begin{aligned}
e^{r f\left(\gamma_{x, y}(t)\right)} & \leq t e^{r f(x)}+(1-t) e^{r f(y)} \\
& \leq t e^{r \lambda}+(1-t) e^{r \lambda}
\end{aligned}
$$

Equivalently,

$$
e^{r f\left(\gamma_{x, y}(t)\right)} \leq e^{r \lambda}
$$

or

$$
f\left(\gamma_{x, y}(t)\right) \leq \lambda
$$

Therefore, $\gamma_{x, y}(t) \in S_{\lambda}$ for all $t \in[0,1]$, and the result is proved.

\section{Geodesic $r$-preinvexity and differentiability}

In this section, we discuss property and condition (say condition $(C)$ ) introduced by Barani and Pouryayevali [12] on the function $\eta: M \times M \rightarrow T M$, which will be used in the subsequent analysis.

Pini [10] define the following property.

Definition 4.1 Let $M$ be a Riemannian manifold and $\gamma:[0,1] \rightarrow M$ be a curve on $M$ such that $\gamma_{x, y}(0)=y$ and $\gamma_{x, y}(1)=x$. Then $\gamma_{x, y}$ is said to possess the property $(\mathrm{P})$ with respect to $y, x \in M$ if

$$
\gamma_{x, y}^{\prime}(s)(t-s)=\eta\left(\gamma_{x, y}(t), \gamma_{x, y}(s)\right)
$$

for all $s, t \in[0,1]$.

Pini [10] also proved the following conditions as follows:

$$
\begin{aligned}
& \left(\mathrm{C}_{1}\right) \quad P_{s, \gamma_{x, y}}^{0}\left[\eta\left(y, \gamma_{x, y(s)}\right)\right]=-s \eta(x, y), \\
& \left(\mathrm{C}_{2}\right) \quad P_{s, \gamma_{x, y}}^{0}\left[\eta\left(x, \gamma_{x, y(s)}\right)\right]=(1-s) \eta(x, y)
\end{aligned}
$$

for all $s \in[0,1]$, which taken together are called condition $(C)$.

Theorem 4.1 Let $M$ be a Riemannian manifold and $S$ be an open subset of $M$ which is a geodesic invex set with respect to $\eta: M \times M \rightarrow$ TM. Let $f: S \rightarrow R$ be a differentiable and geodesic r-preinvex function on $S$. Then $f$ is an $r$-invex function on $S$.

Proof Since $S$ is a geodesic invex set with respect to $\eta$, then for all $x, y \in S$, there exists a unique geodesic $\gamma_{x, y}(0)=y, \gamma_{x, y}^{\prime}(0)=\eta(x, y), \gamma_{x, y}(t) \in S$ for all $t \in[0,1]$. By the differentia- 
bility of $f$ at $y \in M$, we have

$$
d f_{y}(\eta(x, y))=\lim _{t \rightarrow 0} \frac{1}{t}\left[f\left(\gamma_{x, y}(t)\right)-f(y)\right]
$$

and so

$$
f(y)+d f_{y}(\eta(x, y)) t+O^{2}(t)=f\left(\gamma_{x, y}(t)\right) .
$$

But $f$ is geodesic $r$-preinvex for $t \in(0,1]$, and we have

$$
f(y)+d f_{y}(\eta(x, y)) t+O^{2}(t) \leq \log \left(t e^{r f(x)}+(1-t) e^{r f(y)}\right)^{\frac{1}{r}}
$$

or

$$
e^{r f(y)+r d f_{y}(\eta(x, y)) t+r o^{2}(t)}-e^{r f(y)} \leq t\left(e^{r f(x)}-e^{r f(y)}\right) .
$$

Dividing by $t$ and taking the limit $t \rightarrow 0$, we get

$$
e^{r f(y)} d f_{y}(\eta(x, y)) \leq \frac{1}{r}\left(e^{r f(x)}-e^{r f(y)}\right) .
$$

Hence, $f$ is an $r$-invex function on $S$.

Theorem 4.2 Let $M$ be a Riemannian manifold and $S$ be an open subset of $M$, which is a geodesic invex set with respect to $\eta: M \times M \rightarrow T M$. Let $f: S \rightarrow R$ be a differentiable function, $\eta$ satisfies the condition (C), then $f$ is geodesic $r$-preinvex on $S$ iff is $r$-invex on $S$.

Proof We know that for a geodesic invex set with respect to $\eta$ for every $x, y \in S$, there exists a unique geodesic $\gamma_{x, y}:[0,1] \rightarrow M$ such that $\gamma_{x, y}(0)=y, \gamma_{x, y}^{\prime}(0)=\eta(x, y), \gamma_{x, y}(t) \in S$, for all $t \in[0,1]$.

Fix $t \in[0,1]$ and set $\bar{x}=\gamma_{x, y}(t)$, then by geodesic $r$-invexity of $f$ on $S$, we have

$$
\begin{aligned}
& \frac{1}{r} e^{r f(x)}-\frac{1}{r} e^{r f(\bar{x})} \geq e^{r f(\bar{x})} d f_{\bar{x}}(\eta(x, \bar{x})), \\
& \frac{1}{r} e^{r f(y)}-\frac{1}{r} e^{r f(\bar{x})} \geq e^{r f(\bar{x})} d f_{\bar{x}}(\eta(y, \bar{x})) .
\end{aligned}
$$

On multiplying (1) by $t$ and (2) by $(1-t)$, respectively, and then adding we get

$$
t \frac{1}{r} e^{r f(x)}+(1-t) \frac{1}{r} e^{r f(y)}-\frac{1}{r} e^{r f(\bar{x})} \geq e^{r f(\bar{x})} d f_{\bar{x}}[t \eta(x, \bar{x})+(1-t) \eta(y, \bar{x})] .
$$

By the condition (C), we have

$$
t \eta(x, \bar{x})+(1-t) \eta(y, \bar{x})=t(1-t) P_{0, \gamma}^{t}[\eta(x, y)]-(1-t) t P_{0, \gamma}^{t}[\eta(x, y)]=0 .
$$

This together with (3) implies

$$
t e^{r f(x)}+(1-t) e^{r f(y)} \geq e^{r f(\bar{x})}
$$


or

$$
f(\bar{x}) \geq \log \left(t e^{r f(x)}+(1-t) e^{r f(y)}\right)^{\frac{1}{r}}
$$

Hence, $f$ is geodesic $r$-preinvex on $S$.

\section{Geodesic $r$-preinvexity and semi-continuity}

In this section, we discuss geodesic $r$-preinvexity on Riemannian manifold under proximal subdifferential of a lower semi-continuous function. First, we recall the definition of a proximal subdifferentiable of a function defined on a Riemannian manifold in [12].

Definition 5.1 Let $M$ be a Riemannian manifold and $f: M \rightarrow(-\infty, \infty$ ] be a lower semicontinuous function. A point $\xi \in T_{y} M$ is said to be proximal subgradient of $f$ at $y \in \operatorname{dom}(f)$, if there exist a positive number $\delta$ and $\sigma$ such that

$$
f(x) \geq f(y)+\left\langle\xi, \exp _{y}^{-1} x\right\rangle_{y}-\sigma d^{2}(x, y)
$$

for all $x \in B(y, \delta)$, where $\operatorname{dom} f=\{x \in M: f(x)<\infty\}$. The set of all proximal subgradient of $y \in M$ is denoted by $\partial_{p} f(y)$.

Theorem 5.1 Let $M$ be a Riemannian manifold and $S$ be an open subset of $M$, which is geodesic invex with respect to $\eta: M \times M \rightarrow T M$. Let $f: S \rightarrow R$ be geodesic r-preinvex, if $\bar{x} \in S$ is a local minimum of the problem

\section{(P) Minimize $f(x)$}

subject to $x \in S$,

then $\bar{x}$ is a global minimum of $(\mathrm{P})$.

Proof Let $\bar{x} \in S$ be a local minimum; then there exists a neighborhood $N_{\epsilon}(\bar{x})$ such that

$$
f(\bar{x}) \leq f(x)
$$

for all $x \in S \cap N_{\epsilon}(\bar{x})$.

If $\bar{x}$ is not a global minimum of $f$, then there exists a point $x^{*} \in S$ such that

$$
f\left(x^{*}\right)<f(\bar{x})
$$

or

$$
e^{r f\left(x^{*}\right)}<e^{r f(\bar{x})}
$$

As $S$ is a geodesic invex set with respect to $\eta$, there exists a unique geodesic $\gamma$ such that $\gamma(0)=\bar{x}, \gamma^{\prime}(0)=\eta\left(x^{*}, \bar{x}\right), \gamma(t) \in S$, for all $t \in[0,1]$.

If we choose $\epsilon>0$ such that $d(r(t), \bar{x})<\epsilon$, then $\gamma(t) \in N_{\epsilon}(\bar{x})$. From the geodesic $r$-preinvexity of $f$, we have

$$
f(\gamma(t)) \leq \log \left(t e^{r\left(x^{*}\right)}+(1-t) e^{r(\bar{x})}\right)^{\frac{1}{r}} .
$$


Equivalently, we have

$$
e^{r f(\gamma(t))} \leq t e^{r\left(x^{*}\right)}+(1-t) e^{r(\bar{x})}<t e^{r(\bar{x})}+(1-t) e^{r(\bar{x})}
$$

or

$$
e^{r f(\gamma(t))}<e^{r f(\bar{x})}
$$

or

$$
f(\gamma(t))<f(\bar{x})
$$

for all $t \in(0,1]$. Therefore, for each $\gamma(t) \in S \cap N_{\epsilon}(\bar{x}), f(\gamma(t))<f(\bar{x})$, which is a contradiction to (5). Hence the result.

Theorem 5.2 Let $M$ be a Cartan-Hadamard manifold and $S$ be an open subset of $M$, which is geodesic r-preinvex with respect to $\eta: M \times M \rightarrow T M$ with $\eta(x, y) \neq 0$ for all $x \neq y$. Assume that $f: S \rightarrow(-\infty, \infty]$ is a lower semi-continuous geodesic $r$-preinvex function and $y \in \operatorname{dom}(f), \xi \in \partial_{p} f(y)$. Then there exists a positive number $\delta$ such that

$$
e^{r f(x)}-e^{r f(y)} \geq e^{r f(y)}\langle\xi, \eta(x, y)\rangle_{y}
$$

for all $x \in S \cap B(y, \delta)$.

Proof From the definition of $\partial_{p} f(y)$, there are positive numbers $\delta$ and $\sigma$ such that

$$
f(x) \geq f(y)+\left\langle\xi, \exp _{y}^{-1} x\right\rangle_{y}-\sigma d^{2}(x, y)
$$

for all $x \in B(y, \delta)$.

Now, fix $x \in S \cap B(y, \delta)$. Since $S$ is a geodesic invex set with respect to $\eta$, there exists a unique geodesic $\gamma_{x, y}:[0,1] \rightarrow M$ such that $\gamma_{x, y}(0)=y, \gamma_{x, y}^{\prime}(0)=\eta(x, y), \gamma_{x, y}(t) \in S$, for all $t \in[0,1]$.

Since $M$ is a Cartan-Hadamard manifold, then $\gamma_{x, y}(t)=\exp _{y}(t \eta(x, y))$ for each $t \in[0,1]$ (see [4, p.253]). If we choose $t_{0}=\frac{\delta}{\|\eta(x, y)\|_{y}}$, then $\exp _{y}(t \eta(x, y)) \in S \cap B(y, \delta)$ for all $t \in\left[0, t_{0}\right)$.

From the geodesic $r$-preinvexity of $f$, we get

$$
f\left(\exp _{y}(t \eta(x, y))\right) \leq \log \left(t e^{r f(x)}+(1-t) e^{r f(y)}\right)^{\frac{1}{r}}
$$

or

$$
e^{r f\left(\exp _{y}(t \eta(x, y))\right)} \leq t e^{r f(x)}+(1-t) e^{r f(y)} .
$$

Using (6) for each $t \in\left(0, t_{0}\right)$, we get

$$
\begin{aligned}
f\left(\exp _{y}(t \eta(x, y))\right) & \geq f(y)+\left\langle\xi, \exp _{y}^{-1} \exp _{y}(t \eta(x, y))\right\rangle_{y}-\sigma d^{2}\left(\exp _{y}(t \eta(x, y), y)\right) \\
& =f(y)+\langle\xi, t \eta(x, y)\rangle_{y}-\sigma d^{2}\left(\exp _{y}(t \eta(x, y), y)\right) .
\end{aligned}
$$


Since $M$ is a Cartan-Hadamard manifold, for each $t \in\left(0, t_{0}\right)$, we have

$$
d^{2}\left(\exp _{y}(t \eta(x, y), y)\right)=\|t \eta(x, y)\|_{y}^{2}=t^{2}\|\eta(x, y)\|_{y}^{2}
$$

Thus we have

$$
f\left(\exp _{y}(t \eta(x, y))\right) \geq f(y)+\langle\xi, t \eta(x, y)\rangle_{y}-\sigma t^{2}\|\eta(x, y)\|_{y}^{2}
$$

or

$$
e^{r f\left(\exp _{y}(t \eta(x, y))\right)} \geq e^{r f(y)} e^{\langle\xi, t \eta(x, y)\rangle_{y}-\sigma t^{2}\|\eta(x, y)\|_{y}^{2}} .
$$

Thus from (7) and (8), we have

$$
t e^{r f(x)}+(1-t) e^{r f(y)} \geq e^{r f(y)} e^{\langle\xi, t \eta(x, y)\rangle y-\sigma t^{2}\|\eta(x, y)\|_{y}^{2}} .
$$

By further calculation we arrive at

$$
e^{r f(x)}-e^{r f(y)} \geq e^{r f(y)} \frac{1}{t}\left[e^{\langle\xi, t \eta(x, y)\rangle y-\sigma t^{2}\|\eta(x, y)\|_{y}^{2}}-1\right]
$$

taking the limit $t \rightarrow 0$

$$
e^{r f(x)}-e^{r f(y)} \geq e^{r f(y)}\langle\xi, \eta(x, y)\rangle_{y}
$$

This proves the theorem completely.

\section{Mean value inequality}

In this section, we introduce a mean value inequality for Cartan-Hadamard manifold which is an extension of the result proved by Antczak [17] and Barani and Pouryayevali [12].

Definition 6.1 [12] Let $S$ be a non-empty subset of a Riemannian manifold $M$, which is a geodesic $\eta$-invex set with respect to $\eta: M \times M \rightarrow T M$, and let $x$ and $u$ be two arbitrary points of $S$. Let $\gamma:[0,1] \rightarrow M$ be the unique geodesic such that $\gamma(0)=u, \gamma^{\prime}(0)=\eta(x, u)$, $\gamma(t) \in S$, for all $t \in[0,1]$.

A set $P_{u v}$ is said to be a closed $\eta$-path joining the points $u$ and $v=\gamma(1)$, if

$$
P_{u v}=\{y: y=\gamma(t), t \in[0,1]\} .
$$

An open $\eta$-path joining the point $u$ and $v$ is a set of the form

$$
P_{u v}^{0}=\{y: y=\gamma(t), t \in(0,1)\}
$$

If $u=v$ we set $P_{u v}^{0}=\phi$.

Theorem 6.1 (Mean value inequality) Let $M$ be a Cartan-Hadamard manifold and $S$ be an open subset of $M$, which is a geodesic invex set with respect to $\eta: M \times M \rightarrow$ TM such that 
$\eta(a, b) \neq 0$ for all $a, b \in S, a \neq b$. Let $\gamma_{b, a}(t)=\exp _{a}(t \eta(b, a))$ for every $a, b \in S, t \in[0,1]$ and $c=\gamma_{b, a}(1)$. Then a necessary and sufficient condition for a function $f: S \rightarrow R$ to be geodesic $r$-preinvex is that the inequality

$$
e^{r f(x)} \leq e^{r f(a)}+\frac{e^{r f(b)}-e^{r f(a)}}{\langle\eta(b, a), \eta(b, a)\rangle_{a}}\left\langle\exp _{a}^{-1} x, \eta(b, a)\right\rangle_{a}
$$

is true for all $x \in P_{c a}$.

Proof Let $f: S \rightarrow R$ be a geodesic preinvex function, $a, b \in S$ and $x \in P_{c a}$. If $x=a$ or $x=c$ then (9) is true trivially. If $x \in P_{c a}$, then $x=\exp (\operatorname{t\eta }(b, a))$, for some $t \in(0,1)$. From the geodesic $\eta$-invexity of $S$, we have $x \in S$ and

$$
t=\frac{\left\langle\exp _{a}^{-1} x, \eta(b, a)\right\rangle_{a}}{\langle\eta(b, a), \eta(b, a)\rangle_{a}}
$$

Since $f$ is geodesic preinvex on $S$, it follows that

$$
f(x)=f\left(\exp _{a}(t \eta(b, a))\right) \leq \log \left(t e^{r f(b)}+(1-t) e^{r f(a)}\right)^{\frac{1}{r}}
$$

or

$$
\begin{aligned}
e^{r f(x)} & \leq t e^{r f(b)}+(1-t) e^{r f(a)} \\
& =e^{r f(a)}+t\left(e^{r f(b)}-e^{r f(a)}\right) .
\end{aligned}
$$

Using the value of $t$ we get

$$
e^{r f(x)} \leq e^{r f(a)}+\frac{e^{r f(b)}-e^{r f(a)}}{\langle\eta(b, a), \eta(b, a)\rangle_{a}}\left\langle\exp _{a}^{-1} x, \eta(b, a)\right\rangle_{a} .
$$

For sufficiency suppose that the mean value inequality (9) is true. Let $a, b \in S$ and $x=$ $\exp _{a}(t \eta(b, a))$, for some $t \in[0,1]$. Then $x \in S$, and we have $f(x)=f\left(\exp _{a}(t \eta(b, a))\right)$, from (9)

$$
\begin{aligned}
e^{r f(x)} & \leq e^{r f(a)}+\frac{e^{r f(b)}-e^{r f(a)}}{\langle\eta(b, a), \eta(b, a)\rangle_{a}}\left\langle\exp _{a}^{-1} x, \eta(b, a)\right\rangle_{a} \\
& =e^{r f(a)}+\frac{e^{r f(b)}-e^{r f(a)}}{\langle\eta(b, a), \eta(b, a)\rangle_{a}}\left\langle\exp _{a}^{-1}\left(\exp _{a}(t \eta(b, a))\right), \eta(b, a)\right\rangle_{a} \\
& =t e^{r f(b)}+(1-t) e^{r f(a)}
\end{aligned}
$$

or

$$
f(x) \leq \log \left(t e^{r f(b)}+(1-t) e^{r f(a)}\right)^{\frac{1}{r}} .
$$

Equivalently,

$$
f\left(\exp _{a}(t \eta(b, a))\right) \leq \log \left(t e^{r f(b)}+(1-t) e^{r f(a)}\right)^{\frac{1}{r}},
$$

which shows that $f$ is geodesic $r$-preinvex function on $S$. 


\section{Competing interests}

The authors declare that they have no competing interests.

\section{Authors' contributions}

All authors contributed equally to the writing of this paper. All authors read and approved the final manuscript.

\section{Author details}

${ }^{1}$ Department of Mathematics, University of Tabuk, Tabuk, Saudi Arabia. ${ }^{2}$ Department of Mathematics and Statistics, King Fahd University of Petroleum and Minerals, Dhahran, 31261, Saudi Arabia. ${ }^{3}$ Department of Mathematics, King Abdulaziz University, Jeddah, 21589, Saudi Arabia.

Received: 27 November 2013 Accepted: 24 March 2014 Published: 09 Apr 2014

\section{References}

1. Hanson, MA: On sufficiency of the Kuhn-Tucker conditions. J. Math. Anal. Appl. 80, 545-550 (1981)

2. Ben-Israel, B, Mond, B: What is the invexity. J. Aust. Math. Soc. B 28, 1-9 (1986)

3. Craven, BD: Invex functions and constrained local minima. Bull. Aust. Math. Soc. 24, 357-366 (1981)

4. Jeyakumar, V: Strong and weak invexity in mathematical programming. Math. Oper. Res. 55, 109-125 (1985)

5. Jeyakumar, V, Mond, B: On generalized convex mathematical programming. J. Aust. Math. Soc. B 34, 43-53 (1992)

6. Antczak, T: $r$-Preinvexity and $r$-invexity in mathematical programming. Comput. Math. Appl. 50, 551-566 (2005)

7. Antczak, T: V-r-Invexity in multiobjective programming. J. Appl. Anal. 11, 63-80 (2005)

8. Rapcsak, T: Smooth Nonlinear Optimization in $R^{n}$. Kluwer Academic, Dordrecht (1997)

9. Udriste, C: Convex Functions and Optimization Methods on Riemannian Manifolds. Math. Appl. Kluwer Academic, Dordrecht (1994)

10. Pini, R: Convexity along curves and invexity. Optimization 29, 301-309 (1994)

11. Mititelu, S: Generalized invexity and vector optimization on differential manifolds. Differ. Geom. Dyn. Syst. 3, 21-31 (2001)

12. Barani, A, Pouryayevali, MR: Invex sets and preinvex functions on Riemannian manifolds. J. Math. Anal. Appl. 328, 767-779 (2007)

13. Agarwal, RP, Ahmad, I, lqbal, A, Ali, S: Generalized invex sets and preinvex functions on Riemannian manifolds. Taiwan. J. Math. 16(5), 1719-1732 (2012)

14. Zhou, L-W, Huang, N-J: Roughly geodesic B-invex and optimization problem on Hadamard manifolds. Taiwan. J. Math 17(3), 833-855 (2013)

15. Lang, S: Fundamentals of Differential Geometry. Graduate Texts in Mathematics. Springer, New York (1999)

16. Ferreira, OP, Oliveira, PR: Proximal point algorithm on Riemannian manifolds. Optimization 51, 257-270 (2002)

17. Antczak, T: Mean value in invexity analysis. Nonlinear Anal. 60, 1471-1484 (2005)

10.1186/1029-242X-2014-144

Cite this article as: Khan et al.: Geodesic $r$-preinvex functions on Riemannian manifolds. Journal of Inequalities and Applications 2014, 2014:144

\section{Submit your manuscript to a SpringerOpen ${ }^{\circ}$ journal and benefit from:}

- Convenient online submission

- Rigorous peer review

- Immediate publication on acceptance

Open access: articles freely available online

- High visibility within the field

- Retaining the copyright to your article 